\title{
The HERMES Polarized H\&D Gas Target: 10 Years of Operation
}

\author{
Erhard Steffens \\ Physics Institute, University of Erlangen-Nümberg, 91058 Erlangen, Germany \\ and HERMES Collaboration, DESY, 22603 Hamburg, Germany
}

\begin{abstract}
The design and operation of the polarized hydrogen and deuterium gas target internal to the HERA $27.5 \mathrm{GeV}$ polarized electron ring is described. The target has been employed to measure the spin structure of the nucleon, using beams of electrons and positrons, both polarized and unpolarized. The various components of the target system will be described and the operation summarized. The target was removed in November 2005, after 10 years of successful running. It will be utilized in preparation of the PAX experiment at FAIR (Darmstadt) for spin filtering studies of protons and antiprotons.
\end{abstract}

Keywords: Polarized gas targets, electron storage rings, spin structure of the nucleon PACS: 29.25.Pj, 29.20.Dh, 14.20.Dh

\section{INTRODUCTION}

The HERMES experiment was proposed in 1990 for the study of the spin structure of the nucleon by deep-inelastic scattering of polarized electrons off polarized nucleons. After conditional approval in 1992, the detector was commissioned in 1995 , and first data were taken by means of the polarized ${ }^{3} \mathrm{He}$ target [1]. In 1996, the H\&D target was installed and operated in different configurations till 2005.

In order to achieve a luminosity of $10^{31} / \mathrm{cm}^{2} \mathrm{~s}$, target areal densities of $10^{14}$ atoms $/ \mathrm{cm}^{2}$ are required. The clue to these high densities is the Storage Cell, proposed in 1965 by W. Haeberli [2]. By injecting an atomic beam into a T-shaped tube structure, a subspatial stagnation pressure in the center is produced leading to an enhancement of the areal density by more than 100 times, compared with a polarized jet. The drawbacks of this technique like the presence of walls can be minimized by a careful design of the whole target section to an acceptable level. Apart from the density gain, another advantage of storage cells is that due to the many wall collisions individual atoms have a similar history. By measuring the polarization of a sample beam extracted from the cell, the target polarization as seen by the beam can be predicted with good precision. This is mandatory in the case of a high energy electron beam because no scattering channels with high rate and sufficient polarization sensitivity are available to monitor the polarization of the target.

In order to inhibit recombination and depolarization on the cell walls they have to be coated. A key question was the long-term performance of the coating in an environmint with bunch fields and synchrotron radiation which excludes Teflon. First tests of a room temperature cell coated with Drifilm were performed by a BINP-ANL

\footnotetext{
CP915, Proceedings of the $17^{\text {th }}$ International Spin Physics Symposium, edited by K. Imai, T. Murakami, N. Saito, and K. Tanida
} 2007 American Institute of Physics 978-0-7354-0423-6/07/\$23.00 
collaboration in 1988 in the $2 \mathrm{GeV}$ VEPP-3 electron storage ring at Novosibirsk indicating stable target polarization during a six months period. So Drifilm was the natural choice also for HERMES. During the HERMES run it turned out that on such a surface at $100 \mathrm{~K}$ a renewable water surface is formed with superior quality.

One of the conditions for final approval of HERMES was the achievement of an areal target density of $10^{14} \mathrm{H}$-atoms $/ \mathrm{cm}^{2}$. This density was demonstrated in 1992 with a cooled Teflon-coated cell $250 \mathrm{~mm}$ long and inner diameter of $11 \mathrm{~mm}$ [3]. After the full approval in 1993 the target section was designed and built, and the whole target system was successfully tested in an external test set-up.

\section{OVERVIEW OF HERMES H\&D TARGET}

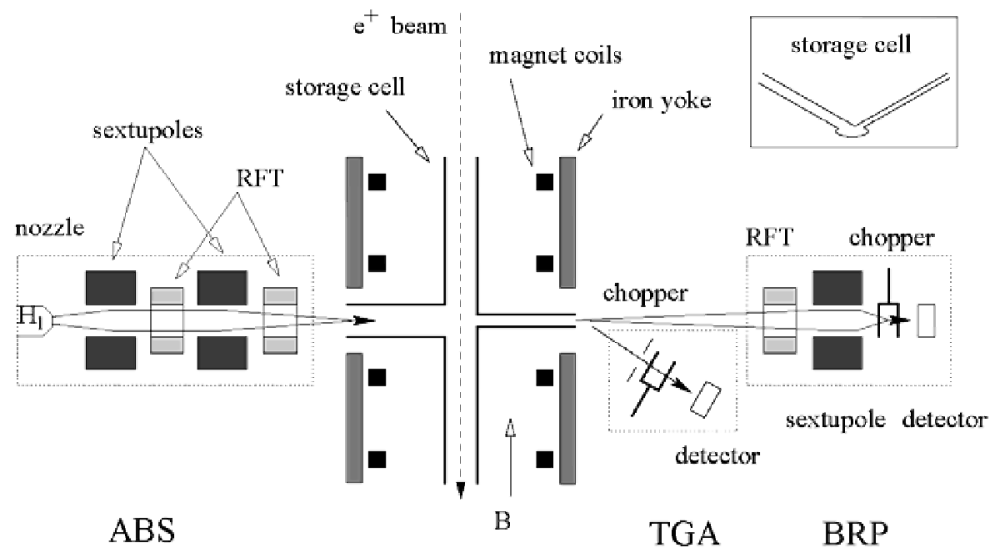

FIGURE 1. Schematic representation of the H\&D target. Left: Atomic Beam Source; Center: Storage cell with beam tube (vertical), feed tube (left) and sample tube (right), the four Helmholtz-type superconducting coils for the longitudinal guide field are indicated; Right: Diagnostics for the sample beam: $\mathrm{BRP}=$ sampling polarimeter, $\mathrm{TGA}=$ gas analyzer. The beam direction of the $\mathrm{e}^{+} / \mathrm{e}^{-}$beam is indicated. The insert shows the spatial arrangement of feed and sample tube as seen from downstream.

The target system (for an overview and detailed references see [4]) is shown schematically in Fig. 1. The requirements imposed by the experiment were

1. Areal density $\mathrm{t}=10^{14} \mathrm{H}$-atoms $/ \mathrm{cm}^{2}$ in two hyperfine structure substates

2. Target cell at $\mathrm{T}=100 \mathrm{~K}$ with coating stable to synchrotron radiation

3. Suppression of Wake Fields and corresponding heating of the cell

4. Suppression of beam-induced depolarization

5. Precise measurement of target polarization independent of beam

Requirements $(1,2)$ seemed to be on safe grounds, $(3,4)$ were studied by simulations only, indicating that the recipe to surround the beam by metal tubes of narrow, slowly varying cross section will work. The full target polarimetry could be tested only with the target installed in the HERA ring. Fortunately, it turned out that despite all the unknowns the required error of $\delta \mathrm{P} / \mathrm{P}=3 \%$ could be achieved for most of the running periods. 


\section{Atomic Beam Source}

The source [5] employs a dissociator with cooled nozzle, a system of permanent sextupole-magnets and rf-transitions in between and behind the magnet system. For hydrogen, a standard rf dissociator at $13.56 \mathrm{MHz}$ with straight Pyrex or Quartz tubes was used, for deuterium a microwave dissociator at $2.45 \mathrm{GHz}$ with superior endurance. For a high atomic fraction in the beam, oxygen 0.1 to 0.3 in Vol. $\%$ is added to the dissociator gas $\mathrm{H}_{2}$ or $\mathrm{D}_{2}$. Water formed in the discharge freezes at the inner surface of the cold nozzle thus gradually reducing the cross section of the nominal $2 \mathrm{~mm}$ nozzle opening which leads to decrease of the target density. After 3-5 days of running, the nozzle is fully recovered by a slight warming up which can be conducted during two fills. In addition, a long-term deterioration of the discharge tubes was observed, probably by erosion due to the discharge and more pronounced for the rf dissociator. This lead to breaking of the glass tubes after a couple of months of continuous operation, bringing the source operation to a complete halt. A new tube had to be installed requiring an access to the experiment of the order of $8 \mathrm{~h}$. Therefore new tubes have been installed preventively to minimize the risk of tube breaking. The particle current of the ABS accepted by the feed tube of the storage cell (see Fig.1) has been determined indirectly by the polarization loss induced via the density-dependent spin exchange process [6]. For hydrogen (2 substates) a flux of $6.5 \cdot 10^{16} / \mathrm{s}$ has been found, for deuterium ( 3 substates) a flux of about $5 \cdot 10^{16} / \mathrm{s}$. The resulting areal densities are given in Tab.1.

\section{Storage Cell Target}

The $400 \mathrm{~mm}$ long storage cell were made of $75 \mathrm{~mm}$ thick Al sheets coated with Drifilm after fabrication. The original elliptical cross section with diameters of 29.8 $\mathrm{mm}$ (hor.) and $9.8 \mathrm{~mm}$ (vert.) were based on the geometry of the collimator system [7] and the requirement that the full length of the cell should not be hit by synchrotron photons scattered from the main (C1) collimator in order to reduce detector background. Later cells of reduced diameter have been tried [4] leading to an optimized cell with diameters $21.0 \mathrm{~mm}$ (hor.) and $8.9 \mathrm{~mm}$ (vert.) which resulted in about $80 \%$ more areal density compared to the original design. The final cell was used from the end of 1999 on.

The cell was suspended between rails cooled by cold helium gas to about $100 \mathrm{~K}$. A homogeneous longitudinal or vertical (transverse) 'strong' magnetic field is applied to decouple electronic and nuclear spins [8]. Its precise strength is chosen in order to minimize beam-induced depolarization.

\section{Target Diagnostics And Evaluation Of The Target Polarization}

As shown in Fig. 1, a sample beam is extracted from the target cell and analyzed by means of two diagnostic devices

- the target polarimeter (BRP) consisting of two rf transitions, a system of sexton-pole magnets and a sensitive atomic beam detector with chopper and particle counting capability for the measurement of substate population $[9,10]$; 
- the analyzer (TGA) detecting the atomic fraction $\alpha^{\mathrm{TGA}}$ of the target gas [11].

The calibration both of the BRP and the TGA can be done solely on the base of measurements which are performed regularly, without relying on assumptions. For the calibration of the BRP, detector signals for all possible combinations of if transition settings both of ABS and BRP are recorded. As they are linearly related to the substate populations via a matrix with coefficients depending on efficiencies of the various if transitions and transmissions through the magnet systems, the matrix can be calculated numerically by means of a Matrix Inversion technique [4, 9]. In this way, both the substate populations $\mathrm{N}_{\mathrm{i}}(\mathrm{i}=1-4$ for $\mathrm{H}, \mathrm{i}=1-6$ for $\mathrm{D})$ of the beam entering the cell, and of the sample beam - called $\mathrm{P}^{\mathrm{BRP}}$ - can be calculated. - For the calibration of the TGA a very elegant self-consistent method [11] has been developed based on the fact that for a constant input of atoms into the cell the sum of atoms plus $2 \mathrm{x}$ the molecules remains constant. This allows for the determination of the relative detection efficiency of atoms $\left(\mathrm{H}^{+}\right)$and molecules $\left(\mathrm{H}_{2}{ }^{+}\right)$of the Quadrupole Mass Spectrometer of the TGA.

The target polarization $\mathrm{P}^{\mathrm{T}}$ is then calculated using

$$
\mathrm{P}^{\mathrm{T}}=\alpha_{\mathrm{o}} \alpha_{\mathrm{r}} \mathrm{P}_{\mathrm{a}}+\alpha_{\mathrm{o}}\left(1-\alpha_{\mathrm{r}}\right) \beta \mathrm{P}_{\mathrm{a}}
$$

Here $\mathrm{P}^{\mathrm{T}}$ denotes the polarization $(\mathrm{z}=$ vector, $\mathrm{zz}=$ tensor) of the nuclei as seen by the beam, i.e. density weighted averages, $\alpha_{\mathrm{o}}$ and $\alpha_{\mathrm{r}}$ the atomic fractions due to dilution by the background gas and by recombination in the cell, respectively. $P_{a}$ is the polarization of atoms in the cell, and $\beta$ the fraction of nuclear polarization carried by recombined molecules with respect to atoms. By an elaborate analysis $[4,6,9,12]$ of the diffusion of particles within the cell, and of the polarization $\mathrm{P}^{\mathrm{BRP}}$ of the sample beam with respect to the injected one, the quantities $\mathrm{P}^{\mathrm{BRP}}$ and $\alpha^{\mathrm{TGA}}$ measured on the sample beam can be corrected resulting in the true values of $\mathrm{P}_{\mathrm{a}}$ and $\alpha_{\mathrm{r}}$ in the cell including the respective errors. The quantity $\alpha_{0}$ is determined by means of controlled gas inlet of $\mathrm{H}_{2}$ gas.

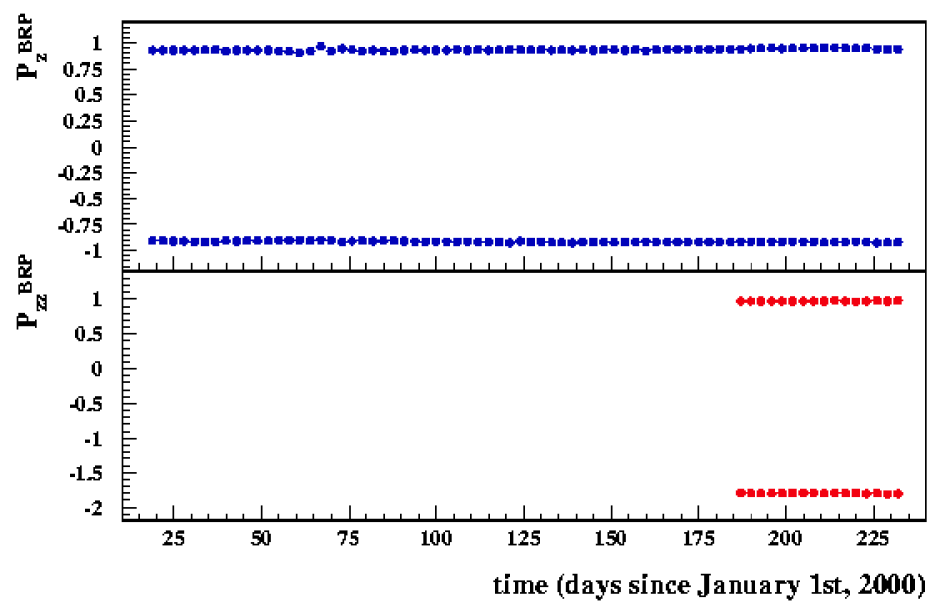

FIGURE 2. Vector (top) and tensor (bottom) polarization during the longitudinal deuterium run in 2000 of about seven months in duration. The points represent three days averages. 


\begin{tabular}{|c|c|c|c|}
\hline & $H_{\|}(1997)$ & $H_{\perp}(2002)$ & $D_{\|}(2000)$ \\
\hline \hline$\alpha_{0}$ & $0.960 \pm 0.010$ & $0.918 \pm 0.032$ & $0.919 \pm 0.026$ \\
\hline$\alpha_{r}$ & $0.945 \pm 0.035$ & $0.979 \pm 0.023$ & $0.997 \pm 0.017$ \\
\hline$P_{z+}$ & $+0.908 \pm 0.016$ & $+0.859 \pm 0.032$ & $+0.927 \pm 0.017$ \\
\hline$P_{z-}$ & $-0.908 \pm 0.016$ & $-0.859 \pm 0.032$ & $-0.915 \pm 0.010$ \\
\hline$-\Delta P_{S E}$ & 0.035 & 0.055 & $\leq 0.001$ \\
\hline$-\Delta P_{W D}$ & 0.02 & 0.055 & $\leq 0.01$ \\
\hline$-\Delta P_{B I}$ & $a b s e n t$ & 0.015 & $a b s e n t$ \\
\hline$P_{+}^{T}$ & $+0.851 \pm 0.031$ & $+0.783 \pm 0.041$ & $+0.851 \pm 0.029$ \\
\hline$P_{-}^{T}$ & $-0.851 \pm 0.031$ & $-0.783 \pm 0.041$ & $-0.840 \pm 0.026$ \\
\hline f $\left(10^{14}\right.$ rucleons/cm $\left.^{2}\right)$ & 0.7 & 1.1 & 2.1 \\
\hline FOM $\left(P^{2} t\right)\left(10^{14} /\right.$ cm $\left.^{2}\right)$ & 0.5 & 0.67 & 1.5 \\
\hline
\end{tabular}

TABLE 1. Overview of the different running periods of the HERMES experiment with H\&D target [4]. The orientation of the target polarization with respect to the electron beam is given. The symbols refer to the notation of equation (1). The $\Delta \mathrm{P}^{\prime} \mathrm{s}$ are the changes of the target polarization due to the various processes occurring in the cell $(\mathrm{SE}=$ spin exchange, $\mathrm{WD}=$ wall depolarization, $\mathrm{BI}=$ beam induced depol.). Average values of target polarization $\mathrm{P}_{\mathrm{T}}$, areal target density $\mathrm{t}$ and figure of merit $\mathrm{FOM}=\mathrm{P}^{2} \cdot \mathrm{t}$ are given in the bottom lines.

\section{CONCLUSIONS}

Average values of the target polarization for the different running periods are shown in Tab.1. Both for the longitudinal $\mathrm{H}$ and $\mathrm{D}$ period, the final target polarization was at

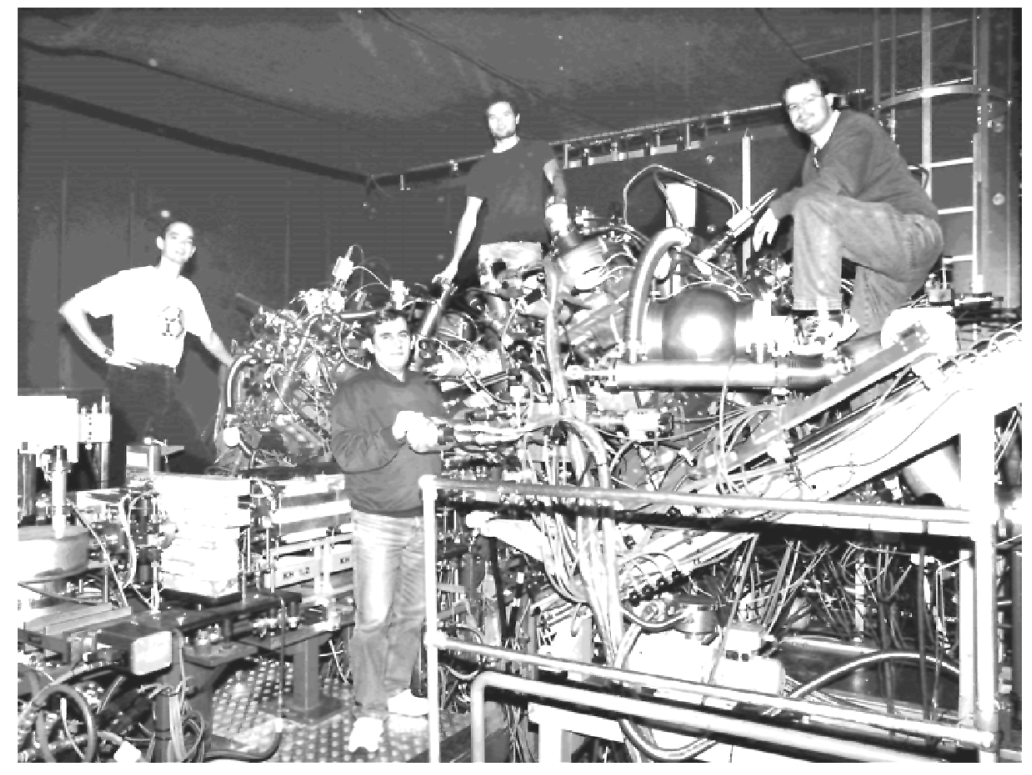

FIGURE 3. Preparations for the removal of the H\&D target system in November 2005. 
$85 \%$ of the possible maximum value. For the transverse target the magnetic guide field was lower in order to limit synchrotron radiation power produced in the target cell. The lower field gave a higher depolarization due to spin exchange. The long-term stability of the target was excellent during most of the time which can be inferred from Fig. 2 covering the 2000 deuterium running period. Depolarization by the harmonics of the bunch field could be suppressed almost completely, even for the transverse $\mathrm{H}$ target for which a new class of narrow-spaced depolarizing resonances exists [13].

Altogether, the target system has worked reliably over a period a ten years in beam position and for most of the calendar time, thanks to the tremendous efforts of numerous scientists, students and technicians. On Nov. 16, 2005 the target was removed from the HERMES detector (see Fig.3) which is operated for the remaining running time in a different configuration including the Recoil Detector [14]. The target is now being modified to serve for preparatory experiments for PAX (Polarized Antiproton eXperiments) [15] at the FAIR facility to be built at GSI (Darmstadt). In this way the target may contribute in future even more to our knowledge on the spin structure of hadrons.

\section{ACKNOWLEDGEMENTS}

The contribution of numerous students, postdocs, senior scientists and technicians to the unprecedented performance of the HERMES target is gratefully acknowledged. Special thanks are due to my colleagues G. Court, P. F.-Dalpiaz, D. Fick, G. Graw, W. Haeberli, R. Milner, B. Povh, K. Rith, to C. Baumgarten, B. Braun, N. Koch, F. Stock, T. Wise and many others who contributed in the initial phase of the target, to P. Lenisa, the target coordinator from 2000 to 2005, to the collaborating institutes Erlangen, Ferrara, Heidelberg, Liverpool, Madison, Marburg, Munich, Beijing and Yerevan, to the funding agencies, in particular BMBF (Germany) and INFN (Italy), and to the HERMES and DESY management.

\section{REFERENCES}

1. D. DeSchepper et al., Nucl. Instr. Meth. A 419(1998) 16

2. W. Haeberli, Proc. $2^{\text {nd }}$ Int. Symp. on Polarization Phenomena of Nucleons, Karlsruhe 1965, Experientia Suppl. Vol. 12, Birkhäuser, Basel, p. 64

3. K. Zapfe et al., Nucl. Instr. Meth. A 368 (1996) 293

4. A. Airapetian et al, Nucl. Instr. and Meth. A540 (2005) 68

5. A. Nass et al., Nucl. Instr. Meth. A505 (2003) 633

6. C. Baumgarten, Doctoral Thesis, Univ. of Munich (2000)

7. HERMES Technical Design Report, DESY-PRC 93/06, 1993

8. E. Steffens and W. Haeberli, Rep. Prog. Phys. 66 (2003) 1887-1935

9. C. Baumgarten et al., Nucl. Instr. Meth. A482 (2002) 606

10. H.-G. Gaul and E. Steffens, Nucl. Instr. Meth. A316 (1992) 297

11. C. Baumgarten et al., Nucl. Instr. Meth. A508 (2003) 268

12. C. Baumgarten et al., Eur. Phys. J. D18 (2002) 37

13. P. Tait, Doctoral Thesis, Univ. of Erlangen (2006)

14. E.C. Aschenauer, Invited Talk, these Proceedings

15. H. Stroeher, Invited Talk, these Proceedings, and www.fz-juelich.de/ikp/pax 